\title{
Research Progress of Gualou Xiebai in the Treatment of Coronary Heart Disease
}

\author{
Hongyu Zhang1, Peng Lei ${ }^{*}$, Shuibao Zhang ${ }^{3}$ \\ 1Shaanxi University of Chinese Medicine, Xianyang 712000, Shaanxi Province, China \\ ${ }^{2}$ Shaanxi Provincial People’s Hospital, Xi'an 710000, Shaanxi Province, China \\ ${ }^{3}$ Puyang People's Hospital of Henan Province, Puyang 457000, Henan Province, China \\ *Corresponding author: Peng Lei, 1005239297@qq.com
}

\begin{abstract}
Gualou Xiebai is a basic prescription for the treatment of angina pectoris in Synopsis of the Golden Chamber. This article is a summary of the theoretical research and clinical application of Gualou Xiebai, in hope to promote the use of Gualou Xiebai in clinical practice.
\end{abstract}

Keywords: Gualou Xiebai; Coronary heart disease; Phlegm, Blood stasis; Clinical application

Publication date: November 2021; Online publication: November 30, 2021

\section{Introduction}

According to the statistical report in Summary of China Cardiovascular Health and Disease Report 2020, the prevalence of cardiovascular diseases in China is still rising, and coronary heart disease is placed first among all cardiovascular diseases ${ }^{[1]}$. Coronary heart disease refers to the heart disease caused by ischemia, hypoxia, or necrosis of the cardiac muscle due to narrowed or occluded lumen in the coronary arteries of the heart, owing to atherosclerosis ${ }^{[2]}$. In consideration of its etiology, pathogenesis, and clinical characteristics, modern traditional Chinese medicine classifies it as "angina pectoris" and "actual heart pain" in traditional Chinese medicine. In Clinical Terms of TCM Diagnosis and Treatment - Diseases Part, issued by the State Bureau of Technical Supervision, angina pectoris (actual heart pain) is incorporated into "heart disease," in which angina pectoris is defined as "due to the stagnation of chest Yang, Yin cold and phlegm retention in the chest, or insufficient heart Qi and lack of motivation, resulting in the obstructions of Qi and blood as well as the loss of blood and nourishment of the heart ${ }^{[3]}$." Taking the stagnation of chest Yang with turbid sputum as the entry point, Zhongjing Zhang introduced Gualou Xiebai, a classic prescription for treating angina and for the effective treatment of coronary heart disease in clinical practice.

\section{Etiology and pathogenesis of angina pectoris}

\subsection{Zhongjing Zhang's theory on the etiology and pathogenesis of treating angina pectoris}

Zhongjing Zhang introduced the term "angina pectoris" in Synopsis of Golden Chamber. In Synopsis of the Golden Chamber, it was mentioned, "If the pulse is too low or too high, the upper Jiao Yang is insufficient, and the lower Jiao Yin is cold; that is, when the chest is arthritic and painful, patients put the blame on its extreme deficiency. Now, Yang deficiency is known to be in the upper Jiao, so those with angina pectoris base on their cold lower Jiao Yin ${ }^{[4]}$." This shows that the causes of angina pectoris are "too high" and "too low." Zhongjing once mentioned that "too high" is due to being filled with evil Qi, "too low" is due to the 
lack of heart Yang, and the implication of "the upper Jiao Yang is insufficient, and the lower Jiao Yin is cold" is that too much is not enough. This is a concise summary illustrating the basic pathogenesis of Zhongjing's theory on the treatment of angina pectoris.

\subsection{Phlegm and blood stasis as the etiology and pathogenesis of coronary heart disease}

Zhongjing Zhang proposed the treatment of coronary heart disease in an article titled Angina Pectoris and Heartache in Synopsis of the Golden Chamber. The original text that goes, "Blood is inhibited and becomes water," shows that blood and body fluid affect and transform into each other in pathological metabolism. Body fluid and blood belong to the essence of Yin; the retention of body fluid and blood is considered as phlegm and blood stasis. Therefore, phlegm dampness and blood stasis are two important manifestations of the Yin essence as disease. They are pathogenic factors, and they transform into each other. $S u W e n-B i$ Lun stated that "the pulse is the house of blood, and it's astringency is heartache," pointing out that blood stasis is an important factor leading to angina pectoris and heartache. Jing Yue Quan Shu - Tan Yu mentioned, "Phlegm is the body fluid of human beings, which is nothing but the transformation of water and grain. If the transformation loses its rightness, it would lead to viscera disease, and body fluid failure; in addition, blood and Qi would become phlegm and saliva ${ }^{[5]}$." If the essence of water and grain is not transported and stored in the body for a long time, it would condense into phlegm; phlegm originates from essence and blood stasis originates from blood ${ }^{[6]}$. Blood stasis can cause phlegm stagnation, and phlegm accumulation in the body can hinder blood. Phlegm and blood stasis can be converted into each other, block meridians in the body, and finally form phlegm and blood stasis. Phlegm and blood stasis are both pathogenic factors in the pathogenesis of coronary heart disease.

\section{Pharmacological studies of traditional Chinese medicine}

Wang Yanping and other researchers analyzed 269 articles with complete prescriptions of Gualou Xiebai in the treatment of cardiovascular diseases. They sorted out and counted the medication frequency of these prescriptions. The statistical results showed that the drugs with the highest frequency in treating cardiovascular diseases were Gualou Xiebai, Allium macrostemon, Salvia miltiorrhiza, and Pinellia ternate ${ }^{[7]}$. Liu Dequan and other researchers collected and collated prescriptions from traditional Chinese medicine masters and deduced 400 prescriptions for chest pain. The top four Chinese herbs were salvia miltiorrhiza, Pinellia ternata, Ligusticum chuanxiong, and Poria cocos ${ }^{[8]}$. The drugs with the highest frequency are discussed in this article.

\subsection{Trichosanthes kirilowii Maxim}

Trichosanthes kirilowii Maxim or Trichosanthes rosthornii Harms is derived from the genus Trichosanthes. It has the functions of clearing heat, removing phlegm, moistening lungs, relieving cough, broadening breasts, and smoothing intestines. It is used for the treatment of chest pain, chest tightness, hypochondriac pain, heaty cough, phlegm turbidness, lung carbuncle, intestinal carbuncle, and other diseases. Its extract or injection has the functions of protecting ischemic myocardium, expanding coronary arteries, and inhibiting platelet aggregation ${ }^{[9]}$.

\subsection{Allii Macrostemonis Bulbus}

Allii Macrostemonis Bulbus, from Liliaceae, is used in diseases such as chest numbness, heartburn, or abdominal distension and pain. The white part of scallions is an edible Chinese herbal medicine, which can inhibit platelet aggregation, reduce lipids in the blood, and prevent atherosclerosis. At the same time, it has 
a wide range of anti-tumor, anti-inflammatory, antioxidant, and antibacterial effect. It can be used clinically to treat asthma, angina, coronary heart disease, acute or chronic bronchitis, hyperlipidemia, myocardial ischemia, and other diseases ${ }^{[10]}$.

\subsection{Pinelliae Rhizoma}

Pinelliae Rhizoma belongs to the Araceae family. It has the functions of reducing phlegm dampness, stopping vomiting, eliminating ruffians, dispersing knot, etc. It is used for wet phlegm, cold phlegm, cough and asthma phlegm, dizziness, palpitation, chest ruffians, depression, and other diseases. In addition, Pinellia Rhizoma has anti-tumor, anti-inflammatory, anti-ulcer, and anti-arrhythmic effect.

\subsection{Salvia miltiorrhiza Bge}

Salvia miltiorrhiza Bge is a labriform plant, which has the effect of promoting blood circulation, removing blood stasis, clearing the heart, relieving pain in menstruation, cooling blood, and eliminating carbuncle. It is used for chest pain, heat pain, hypochondriac pain, accumulation of symptoms, restlessness, and other diseases. The main effects of Salvia miltiorrhiza include inhibition of inflammatory response, scavenging of free radicals, anti-oxidation, lowering blood lipids, regulating calcium metabolism, prevent atherosclerosis, blood vessel dilation, improvement of myocardial ischemia, inhibition of platelet aggregation, anti-thrombosis, and other pharmacological effects ${ }^{[1]}$.

\section{Clinical application}

Gualou Xiebai is one of the three famous prescriptions by Zhongjing Zhang in the treatment of angina pectoris and heartache as stated in Synopsis of the Golden Chamber: Treatment of Chest Pains, Heartaches, Shortness of Qi, and Abnormal Pulse ${ }^{[12]}$. The three prescriptions are Gualou Xiebai Banxia Decoction, Gualou Xiebai Baijiu Decoction, and Zhishi Xiebai Guizhi Decoction. It has been established that the treatment of angina pectoris and heartache is based on warming Yang, dispersing cold, broadening chest, and relieving pain. As a basic prescription for treating chest pain, Gualu Xiebai Baijiu Decoction activates Yang, removes obstruction, and removes phlegm to bring Qi down. The liquor in the prescription can remove obstruction by spicy and transport Yang by warm. Gualou Xiebai Banxia Decoction is added with Pinelliae Rhizoma to dispel phlegm, disperse knot, expel rheum, and downbear counterflow. If chest Yang is sluggish and phlegm stops accumulating, Zhishi Xiebai Guizhi Decoction can be used to free Yang and rid impediment, relieving and eliminating mass. Angina pectoris and heartache, caused by chest Yang depression, phlegm turbidity, and blood stasis, are treated with Gualou Xiebai. Research on the mechanism of action of Gualou Xiebai in modern pharmacology has shown that it can protect cardiomyocytes from myocardial reperfusion injury, inhibit platelet aggregation, as well as prevent thrombosis and myocardial ischemia; thus, it is said to be effective in the clinical treatment of coronary artery disease ${ }^{[13]}$. Zhang Zhongjing clearly put forward that "patients with heart arthralgia has blocked pulse," which is the main pathogenesis of angina pectoris. The syndrome elements of the etiology and pathogenesis of angina pectoris are mainly empirical syndromes such as phlegm-rheum, blood stasis, and Qi stagnation. Therefore, the treatment of angina pectoris and heartache should focus on warming heart Yang, removing blood stasis, and resolving phlegm ${ }^{[14]}$. In actual practice, phlegm turbidity and blood stasis often occur together; hence, the method of dredging Yang and eliminating phlegm as well as the method of promoting blood circulation and removing blood stasis are often used at the same time.

\section{Discussion}

Coronary heart disease seriously affects people's health and brings a heavy economic burden to the society; 
it has become a major public health problem. It is more important to prevent the occurrence of the disease and improve the quality of life of patients. Traditional Chinese medicine has more advantages in solving the "overall" problem of the disease, or some "early" and "follow-up" problems that cannot be solved by western medicine. Beginning from a holistic view and the syndrome differentiation and treatment, traditional Chinese medicine can better target the pathogenesis and treat both symptoms and signs. At the same time, traditional Chinese medicine is easily accepted by patients because of its characteristics: multitarget, multi-channel, pure natural components, and minimal side effects. For coronary heart disease, it may be beneficial to give full play to the early preventive effect of Chinese medicine and the prognosis of Chinese medicine in the later stage. Combined with the advantages of modern western medicine in emergency treatment for acute and severe cases, a complete treatment system of Chinese and western medicine should be established, in order to decrease incidence rate and mortality rate of coronary heart disease in China. It has broad scientific research prospects and has extremely important research value.

\section{Disclosure statement}

The authors declare that there is no conflict of interest.

\section{References}

[1] 2021, 2020 Summary of China Cardiovascular Health and Disease Report. Chinese Circulation Journal, 36(06): 521-545.

[2] Ge J, Xu Y, 2014, Internal Medicine Version 8, People's Medical Publishing House, Beijing.

[3] Zhu W, 1998, Routine Diagnosis and Treatment of Internal Diseases of Traditional Chinese Medicine, Hunan Science and Technology Press, 247.

[4] Qiu M, Sun K, Tao J, et al., 2017, Synopsis of Golden Chamber On the Pathogenesis of Chest Arthralgia "Yangwei Yinxian". Chinese Journal of Basic Medicine in Traditional Chinese Medicine, 23(02): 151-152.

[5] Wang D, 2010, Translation and Annotation of Jing Yue Quan Shu, China Renmin University Press, Beijing.

[6] Xu D, Wu H, 2018, Study on the Effect of Phlegm and Blood Stasis on Coronary Heart Disease. China Journal of Traditional Chinese Medicine and Pharmacy, 33(6): 2503-2506.

[7] Wang Y, Peng D, Liu X, et al., 2016, A Meta-Analysis of Gualouxiebaibanxia Decoction in the Treatment of Chest Paralysis. Liaoning Journal of Traditional Chinese Medicine, 43(10): 2051-2056.

[8] Liu D, Huang F, Tian G, Study on the Compatibility of Gualousan-Allium Macrostemon in the Treatment of Chest Pain Based on Association Rules. Journal of Hubei University of Traditional Chinese Medicine, 23(02): 108-111.

[9] Huang Y, Wang Q, Zhu X, et al., 2019, Effect of Trichosanthes Trichosanthes on Coronary Heart Disease. Chin J Cardiol, 7(23): 18.

[10] Sheng Y, Li M, Guo Y, et al., 2020, Research Progress on Chemical Constituents of Allium Macrostemon and Their Extraction and Isolation. Specialty Research, 42(05): 61-70.

[11] Jiang X, Shi L, 2017, Research Progress of Active Components and Pharmacological Effects of Salvia Miltiorrhiza. Pharmaceutical Research, 36(03): 166-169.

[12] Han, Zhang Z, 2005, Synopsis of the Golden Chamber [He R, He R, eds.], People's Medical Publishing House, Beijing, 31-32. 
[13] Tang S, 3027, Observation on Curative Effect of Gualou Xiebai Decoction in Treating Angina Pectoris of Coronary Heart Disease and its Effect on Vascular Endothelial Function. Shanxi Medical Journal, 46(22): 2786-2789.

[14] Xu W, Hu J, Jiang L, 2017, Analysis of the Original Meaning of Gualou Xiebai in Treating Chest Paralysis in Synopsis of Golden Chamber. China Journal of Traditional Chinese Medicine and Pharmacy, 32(5): 2288-2290. 